\title{
Surfactant Gel Extraction of Gold(III), Palladium(II), Platinum(II), and Lead(II) as Thiourea-complexes
}

\author{
Shoji TagaShira, ${ }^{\dagger}$ Sakurako Kimoto, Koji Nozaki, and Yoshiko MuraKami \\ Department of Chemistry, Graduate School of Science and Engineering, Yamaguchi University, \\ Yoshida, Yamaguchi 753-8512, Japan
}

\begin{abstract}
Anionic surfactant solutions of sodium dodecylbenzenesulfonate (SDBS) and sodium dodecylsulfate (SDS) separate into two phases through cooling of the solution and the addition of salts. Thiourea (TU) reacts with many metals to form cationic complexes of $\left[\mathrm{M}(\mathrm{TU})_{\mathrm{n}}\right]^{\mathrm{m}+}$; some of them extract into the SDBS phase. The separation was examined for lead and tin in commercially purchased solders. More than $95 \%$ lead was extracted into the SDBS phase, while tin remained in the aqueous phase. An abnormal decrease in extractability was observed for the $\left[\mathrm{M}(\mathrm{TU})_{\mathrm{n}}\right]^{\mathrm{m}+}$ of noble metal complexes at low metal concentrations. The surfactant phases of SDBS and SDS were confirmed as lamellar structures using small angle $\mathrm{X}$-ray scattering (SAXS), and the stability and rigidity of this structure influenced the extractability of the $\left[\mathrm{Au}(\mathrm{TU})_{\mathrm{n}}\right]^{3+}$ complex. The separation of gold(III) and palladium(II) was examined for the SDS system.
\end{abstract}

(Received February 20, 2009; Accepted March 23, 2009; Published May 10, 2009)

\section{Introduction}

Interest has been shown in the separation of metal ions via extraction methods using a solid or liquid surfactant as the extraction medium. For this kind of method, phase separation due to changing the temperature and/or the addition of a saltingout reagent has been commonly used for the extraction, purification, and preconcentration of metals. ${ }^{1-5}$ A micellar solution of anionic surfactants shows phase separation when the solution is cooled below the Krafft point temperature. These are the surfactant-depleted (aqueous phase) and surfactant-rich (surfactant phase) phases. Most of the separated surfactant phase is a gel-like charged solid that has the ability to bind an oppositely charged solute. In this case, an electrostatic attractive force is the operative phenomenon for the formation of ionpairs, and a hydrophobic domain is the extraction medium. In order to remove metal ions from water, the formation of stable ionic complexes is used to obtain high efficiency and selectivity. The separation of metals is based on the stability of ionic complexes and ion-pairs with counter ions; therefore, the extractability of metals is influenced by the ligand concentration and species of the counter ion, which is commonly the surfactant. This mechanism is the same as for traditional liquidliquid extraction systems using organic solvents.

We previously reported that an unexpected decrease in extractability for the complexes was observed at low metal concentrations for the ion-pair extraction systems of dodecylsulfate ions (DS-) as the anionic surfactant and cationic metal-ammine complexes. ${ }^{6}$ Thiourea, as well as ammonia, is an important chemical substance because it has a large number of industrial utilizations, and is economically easy to obtain. Thiourea forms simple coordination-type complexes with numerous heavy metals, most of which are colorless and soluble

$\dagger$ To whom correspondence should be addressed.

E-mail: taga@yamaguchi-u.ac.jp in water. $^{7}$ Some colored complexes, such as those of palladium(II) and osmium(II), have been used as reagents for spectrophotometric determination in acidic solution. Thiourea has mainly been used as a masking reagent for analytical purposes. A large number of thiourea derivatives ${ }^{8,9}$ have been developed for metal extraction; however, there has been little interest in direct extraction with thiourea because it lacks selectivity. A basic study of surfactant gel extraction using thiourea can be applied to related compounds.

This study examined the extraction of thiourea complexes in anionic surfactant systems. Based on the dependence of the ligand or metal concentration on the extractability, the extractions were examined for the separation of lead(II) and tin(II) using sodium dodecylbenzenesulfonate (SDBS) and of palladium(II) and gold(III) using sodium dodecylsulfate (SDS).

\section{Experimental}

\section{Reagents and solutions}

A $1.00 \times 10^{-2} \mathrm{~mol} \mathrm{dm}^{-3}$ stock solution of lead(II) was prepared by dissolving weighed amounts of lead(II) chloride (Katayama Chemicals, Tokyo) and then diluting to $250 \mathrm{~cm}^{3}$ with deionized water. A $0.10 \mathrm{~mol} \mathrm{dm}^{-3}$ stock solution of tin(II) was prepared by dissolving weighed amounts of tin(II) chloride (Kanto Chemicals, Tokyo) in $10 \mathrm{~cm}^{3}$ of concentrated hydrochloric acid, and then diluting to $100 \mathrm{~cm}^{3}$ with deionized water. A $1.02 \times$ $10^{-2} \mathrm{~mol} \mathrm{dm}^{-3}$ stock solution of gold(III) was prepared by dissolving weighed amounts of gold(III)chloride hydrochloric acid (Merck, Germany) in $2 \mathrm{~cm}^{3}$ of concentrated hydrochloric acid, and then diluting to $250 \mathrm{~cm}^{3}$ with deionized water. A 1.00 $\times 10^{-2} \mathrm{~mol} \mathrm{dm}^{-3}$ stock solution of platinum(II) ${ }^{10}$ was prepared by dissolving weighed amounts of potassium tetrachloroplatinate(II) (Kanto Chemicals) in $4 \mathrm{~cm}^{3}$ of concentrated hydrochloric acid, and then diluting to $100 \mathrm{~cm}^{3}$ with deionized water. A $5.64 \times 10^{-2}$ mol $\mathrm{dm}^{-3}$ stock solution of palladium(II) was prepared by dissolving weighed amounts of palladium(II) chloride (Kanto 
Table 1 Composition and extraciton results of solder

\begin{tabular}{lcccccc}
\hline \multirow{2}{*}{ Sample } & \multicolumn{2}{c}{ Composition, $\%^{\mathrm{a}}$} & & \multicolumn{2}{c}{ Percent extraction, $\%^{\mathrm{b}}$} \\
\cline { 2 - 3 } \cline { 5 - 6 } & Lead & Tin & & Lead & Tin \\
\hline Taiyo & $49.3 \pm 0.1$ & $50.7 \pm 0.1$ & & $95.9 \pm 1.4$ & n.d. \\
Topman & $54.0 \pm 0.3$ & $46.0 \pm 0.3$ & & $97.4 \pm 0.9$ & n.d. \\
\hline
\end{tabular}

a. Average \pm SD (3 samples).

b. Average $\pm \mathrm{SD}$ (4 samples).

Chemicals) in $4 \mathrm{~cm}^{3}$ of concentrated hydrochloric acid, and then diluting to $100 \mathrm{~cm}^{3}$ with deionized water. The $10 \%$ (w/v) SDBS (hard type; Tokyo Kasei, Tokyo) and 10\% (w/v) SDS (Wako Pure Chemicals, Tokyo) solutions, acting as anionic surfactants, and a salting-out reagent of $20 \%$ (w/v) sodium chloride (Kanto Chemicals) solution were prepared by dissolving these reagents in deionized water. A $1.00 \mathrm{~mol} \mathrm{dm} \mathrm{dm}^{-3}$ thiourea (TU) solution used as the chelating agent was prepared by dissolving $19.03 \mathrm{~g}$ of thiourea (Wako Pure Chemicals) in $250 \mathrm{~cm}^{3}$ of deionized water. All chemicals were of analytical grade and dissolved in deionized water.

\section{Apparatus}

The metal concentration was determined using a Varian Liberty Series II inductively coupled plasma atomic emission spectrometer (ICP-AES). All of the $\mathrm{pH}$ measurements were performed using a Horiba F-22 $\mathrm{pH}$ meter in combination with a glass electrode. A Hitachi Himac CF7D2 centrifuge was used for phase separation. A small angle X-ray scattering (SAXS) measurement was performed with a Mac Science powder X-ray diffractometer using copper $K_{\alpha}$ radiation. The concentration of dodecylbenzenesulfonate anions (DBS-) was determined through total organic carbon (TOC) measurements using a Shimadzu TOC-5000A.

\section{Procedure}

Separation of lead(II) and tin(II) in the SDBS system. A solution containing lead(II) and tin(II) was transferred to a 10$\mathrm{cm}^{3}$ centrifuge tube with a graduation line. After adding $2 \mathrm{~cm}^{3}$ each of $0.50 \mathrm{~mol} \mathrm{dm}^{-3} \mathrm{HCl}, 1.0 \mathrm{~mol} \mathrm{dm}{ }^{-3}$ thiourea, and $10 \%(\mathrm{w} / \mathrm{v})$ SDBS, the solution was shaken to form the ion-pair. Then, $1 \mathrm{~cm}^{3}$ of $20 \%(\mathrm{w} / \mathrm{v})$ sodium chloride solution was added, and the mixture was diluted to the marked line with deionized water. After cooling the solution in an ice-water bath and centrifuging (4000 rpm, $5 \mathrm{~min}, 0^{\circ} \mathrm{C}$ ), the aqueous and SDBS phases were separated.

Separation of gold(III) and palladium(II) in the SDS system. A solution containing gold(III) and palladium(II) was transferred to a $10-\mathrm{cm}^{3}$ centrifuge tube with a graduation line. After adding $2 \mathrm{~cm}^{3}$ each of $0.50 \mathrm{~mol} \mathrm{dm}{ }^{-3} \mathrm{HCl}, 5.0 \times 10^{-3} \mathrm{~mol} \mathrm{dm}^{-3}$ thiourea, and $1 \%(\mathrm{w} / \mathrm{v})$ SDS, the solution was shaken to form an ion-pair. Then, $1 \mathrm{~cm}^{3}$ of a $20 \%$ (w/v) sodium chloride solution was added, and the mixture was diluted to the marked line with deionized water. After cooling and centrifuging the solution, palladium(II) was extracted in the SDS phase as a thioureacomplex, while gold(III) remained in the aqueous phase.

Separation of lead and tin in solder. A pretreatment of the sample was carried out as follows. Two hundred fifty milligrams of solder (Taiyo Electric or Topman, Japan) were weighed out and decomposed with $50 \mathrm{~cm}^{3}$ aqua regia. The solution was gently heated and completely dissolved. After being left standing to cool, it was diluted with water to $1 \mathrm{dm}^{3}$ and filtered with No. 5 B filter paper (Toyo Roshi, Japan). The

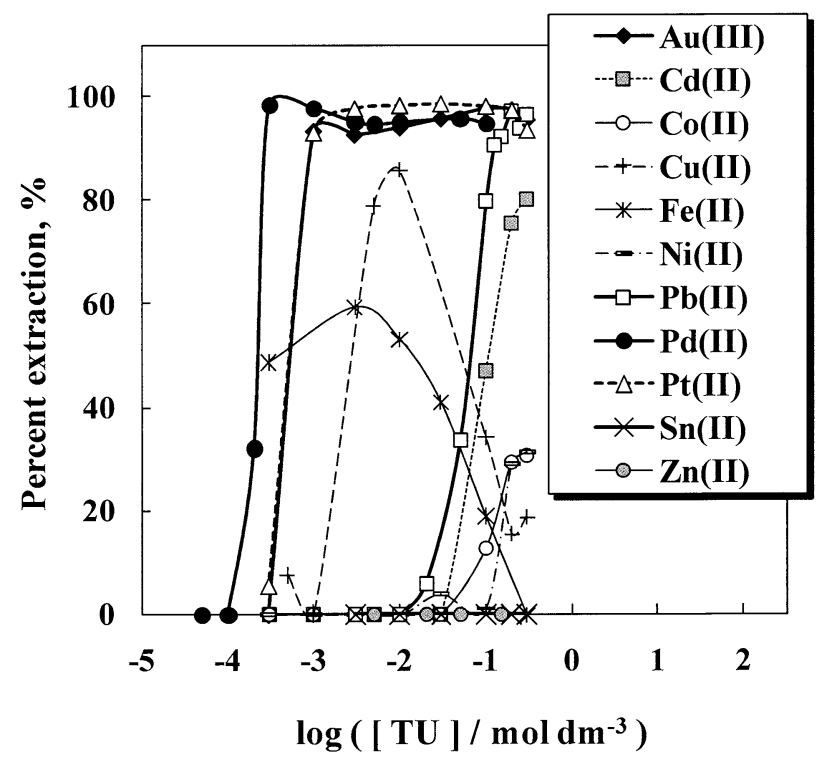

Fig. 1 Effect of the thiourea concentration on the percent extraction of thiourea-complexes. [Metal] $=1.0 \times 10^{-4} \mathrm{~mol} \mathrm{dm}^{-3},[\mathrm{SDBS}]=2 \%$ $(\mathrm{w} / \mathrm{v}),[\mathrm{NaCl}]=2 \%(\mathrm{w} / \mathrm{v}) ; \mathrm{pH} 1$.

metal concentration was determined using ICP-AES. The compositions of the solders consisting of lead and tin are listed in Table 1. Separation of these metals was performed by extraction with thiourea. The results of the separation are also listed in Table 1. More than $95 \%$ lead could be extracted into the SDBS phase, while tin remained in the aqueous phase.

\section{Results and Discussion}

\section{Extraction of metal complexes into the SDBS phase}

The effect of the thiourea concentration on the extraction was studied from $3.0 \times 10^{-4}$ to $0.30 \mathrm{~mol} \mathrm{dm}{ }^{-3}$. The obtained results are shown in Fig. 1. The noble metals gold(III), palladium(II), and platinum(II) could be extracted at low concentrations because they formed stable complexes with thiourea. Some base metals, such as lead(II) and cadmium(II), could be quantitatively extracted at high concentrations. Tin(II) and zinc(II) were not extracted over the entire thiourea concentration range studied.

The effect of the $\mathrm{pH}$ on extraction was studied. The obtained results are shown in Fig. 2. Gold(III) and lead(II) were extracted as thiourea-complexes. Tin(II) seemed to be extracted at a $\mathrm{pH}$ of over 2; however, it formed a precipitation of hydroxide over $\mathrm{pH}$ 1.7. In order to avoid metal hydroxides, subsequent studies were performed at $\mathrm{pH} 1$.

The effect of the SDBS concentration on the extractability of lead(II) was studied from 0 to $3 \%(\mathrm{w} / \mathrm{v})$, and quantitative extraction could be seen over $0.2 \%(\mathrm{w} / \mathrm{v})$. Thus, subsequent studies were performed at $2 \%(\mathrm{w} / \mathrm{v})$.

The effect of the sodium chloride concentration was studied from 0 to $3 \%(\mathrm{w} / \mathrm{v})$. Lead(II) was quantitatively extracted over the entire tested sodium chloride concentration range. Although phase separation occurred through solution cooling alone, it was sometime incomplete without the addition of a salting-out reagent. Subsequent studies were performed at $2 \%(\mathrm{w} / \mathrm{v})$.

\section{Structure of SDBS phase}

The anionic surfactant of SDBS deposits as a gel-like solid 


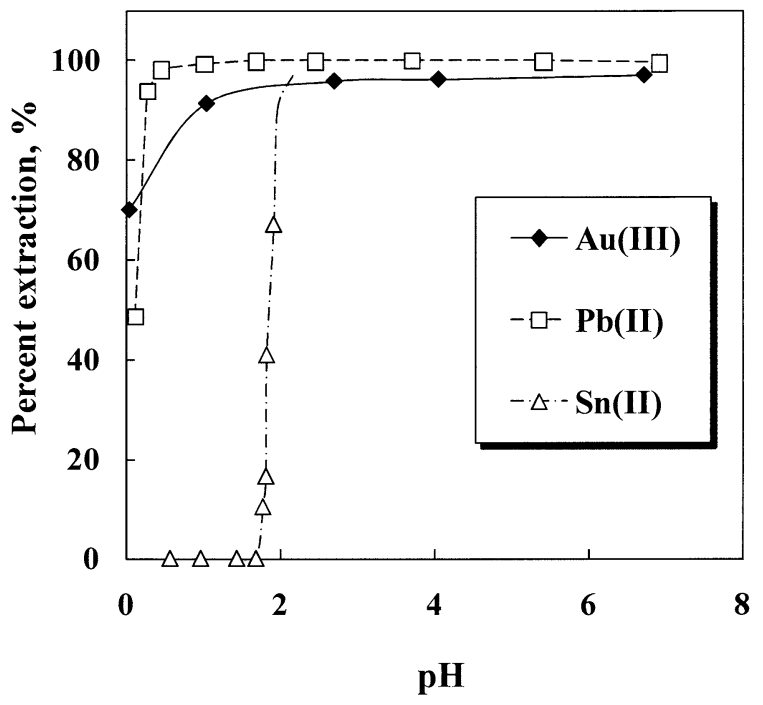

Fig. 2 Effect of the $\mathrm{pH}$ on the percent extraction of thioureacomplexes. $[$ Metal $]=1.0 \times 10^{-4} \mathrm{~mol} \mathrm{dm}^{-3},[\mathrm{TU}]=0.20 \mathrm{~mol} \mathrm{dm}^{-3}$, $[\mathrm{SDBS}]=2 \%(\mathrm{w} / \mathrm{v}),[\mathrm{NaCl}]=2 \%(\mathrm{w} / \mathrm{v})$.

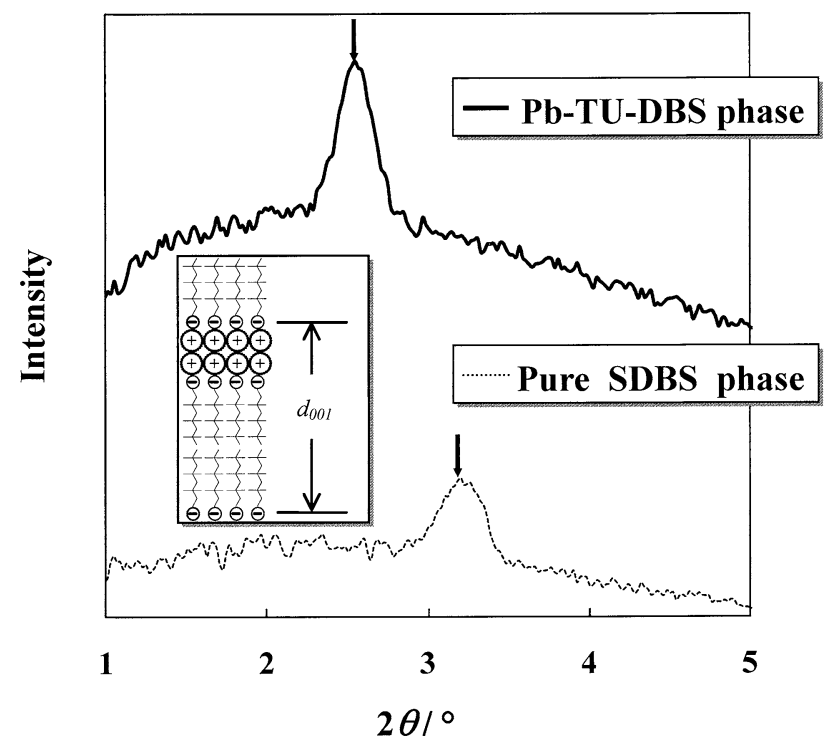

Fig. 3 Resulting patterns of pure SDBS and Pb-TU-DBS phases with SAXS. The inserted figure is the lamellar structure.

from an aqueous solution. It has an amphiphilic nature and frequently appears to have a lamellar structure with alternating head-to-head (hydrophilic layer) and tail-to-tail (hydrophobic layer) arrangements. ${ }^{11}$ Between the heads of the hydrophilic layer, there are $\mathrm{Na}^{+}$counter ions and water molecules. Due to $\left[\mathrm{Pb}(\mathrm{TU})_{\mathrm{n}}\right]^{2+}$ extraction, the $\mathrm{Na}^{+}$ions are replaced by this cationic complex. The ion-exchange of $\mathrm{Na}^{+}$with $\left[\mathrm{Pb}(\mathrm{TU})_{\mathrm{n}}\right]^{2+}$ changes the thickness of the hydrophilic layer. SAXS is useful in estimating the interlayer distance, which is designated as $d_{001}$ in Fig. 3. Figure 3 shows the diffraction patterns obtained with the pure SDBS phase and the phase containing the $\left[\mathrm{Pb}(\mathrm{TU})_{\mathrm{n}}\right]^{2+}$ complex. When the extraction was performed at the molar ratio of $1: 2=\mathrm{Pb}$ :SDBS without a salting-out reagent, all of the sodium ions in SDBS were completely replaced by $\left[\mathrm{Pb}(\mathrm{TU})_{\mathrm{n}}\right]^{2+}$; this phase is abbreviated as the $\mathrm{Pb}$-TU-DBS phase. The reflection peak was observed at $2 \theta=3.20^{\circ}$ for the pure SDBS

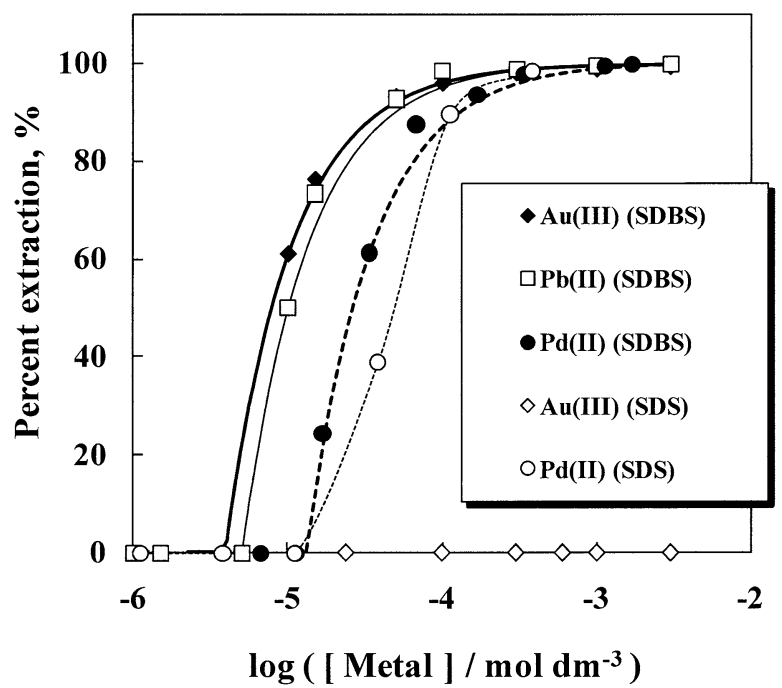

Fig. 4 Effect of the metal concentration on the extraction by SDBS and SDS. Lines for SDBS are theoretical curves according to Eqs. (3) and (4). $[\mathrm{TU}]=0.20 \mathrm{~mol} \mathrm{dm}^{-3}$ for lead(II), $1.0 \times 10^{-3} \mathrm{~mol} \mathrm{dm}^{-3}$ for gold(III) and palladium(II). [SDBS] $=2 \%(\mathrm{w} / \mathrm{v}),[\mathrm{SDS}]=0.2 \%$ $(\mathrm{w} / \mathrm{v}),[\mathrm{NaCl}]=2 \%(\mathrm{w} / \mathrm{v}) ; \mathrm{pH} 1$.

phase and $2 \theta=2.54^{\circ}$ for the $\mathrm{Pb}$-TU-DBS phase. These peaks showed characteristics of the lamellar structure. The interlayer distances were calculated with Bragg's equation using the obtained angles,

$$
2 d \sin \theta=n \lambda .
$$

The interlayer distances were 2.76 and $3.49 \mathrm{~nm}$ for the SDBS and $\mathrm{Pb}-\mathrm{TU}-\mathrm{DBS}$ phases, respectively. This distance change supported the intercalation of $\left[\mathrm{Pb}(\mathrm{TU})_{\mathrm{n}}\right]^{2+}$ into the hydrophilic layer of the surfactant phase.

Dependence of the metal concentration on the percent extractability The dependence of the metal concentration on the extractability is shown in Fig. 4. The extractability of gold(III), lead(II) and palladium(II) in the SDBS system decreased to under $1.0 \times 10^{-4} \mathrm{~mol} \mathrm{dm}{ }^{-3}$ metal concentration. These decreases can be explained by the unique solubility product of ion-pairs between anionic surfactants and cationic-complexes. ${ }^{6}$

In the presence of a surfactant gel, the concentration of monomers ([DBS- $]_{\mathrm{aq}}$ ) was independent from the initial surfactant concentrations and constant in the aqueous phase. The solubility product of the ion-pair $K_{\mathrm{sp}}$ is defined as follows:

$$
K_{\mathrm{sp}}=\left[\mathrm{M}(\mathrm{TU})_{\mathrm{n}}^{2+}\right]_{\mathrm{aq}}\left(\left[\mathrm{DBS}^{-}\right]_{\mathrm{aq}}\right)^{2},
$$

where $\left[\mathrm{M}(\mathrm{TU})_{\mathrm{n}}{ }^{2+}\right]_{\mathrm{aq}}$ denotes the equilibrium concentrations of the metal thiourea-complex in the aqueous phase. The concentration of $\left[\mathrm{DBS}^{-}\right]_{\mathrm{aq}}$ was obtained by TOC measurements in the aqueous phase $\left(\left[\mathrm{DBS}^{-}\right]_{\mathrm{aq}}=1.21 \times 10^{-3} \mathrm{~mol} \mathrm{dm}^{-3}\right)$. The equations related to the percent extraction $E(\%)$ and solubility product are

$$
E(\%)=\left\{1-\left(K_{\mathrm{sp}} /\left(\left[\mathrm{DBS}^{-}\right]_{\mathrm{aq}}\right)^{2}[\mathrm{M}]_{\mathrm{ini}}\right)\right\} \times 100 .
$$

The values of $-\log K_{\mathrm{sp}}$ were 11.3 and $10.8\left(\mathrm{~mol} \mathrm{dm}^{-3}\right)^{3}$ for lead(II) and palladium(II), respectively. The value of 14.2 (mol $\left.\mathrm{dm}^{-3}\right)^{4}$ for gold(III) was obtained using Eq. (4).

Pure and dry surfactants can be made to crystallize as a 
Table 2 Separation of gold(III) and palladium(II) with SDS (percent extraction, \% $\%^{a}$ )

\begin{tabular}{cc}
\hline Gold(III) & Palladium(II) \\
\hline n.d. & $92.2 \pm 2.4$ \\
\hline
\end{tabular}

a. Average $\pm \mathrm{SD}$ (4 samples).

lamellar structure relatively easily. The energy of the surfactant crystal is determined primarily by the chemical structure of the molecules. For example, the crystallizing energy of $n$-alkyl sulfate surfactants is greater than that for branched chains due to the more compact and ordered packing structures available to the straight-chain materials. ${ }^{11}$ The SDBS used in this study was a branched surfactant (hard type). Therefore, the extraction of metals was examined with the $n$-alkyl sulfate of SDS. As can be seen in Fig. 4, the Pd-TU-complex $\left(\left[\mathrm{Pd}(\mathrm{TU})_{\mathrm{n}}\right]^{2+}\right)$ was extracted into both the SDBS and SDS phases. The extraction was examined at $1.0 \times 10^{-3}$ (Fig. 4) or $0.20 \mathrm{~mol} \mathrm{dm}^{-3}$ thiourea concentration; however, gold(III) was not extracted into the SDS phase. The separation of palladium(II) and gold(III) was examined in the SDS system. The results of the separation are given in Table 2. More than $90 \%$ palladium(II) could be extracted into the SDS phase, while gold(III) remained in the aqueous phase.

The exact mechanism for the extraction of metal complexes has been unknown in surfactant systems. The complexes of bivalent metals can produce ion-pairs with two $\mathrm{DS}^{-}$and form a lamellar structure. For gold(III), Eq. (2) can be rewritten as

$$
K_{\mathrm{sp}}=\left[\mathrm{M}(\mathrm{TU})_{\mathrm{n}}{ }^{3+}\right]_{\mathrm{aq}}\left(\left[\mathrm{DBS}^{-}\right]_{\mathrm{aq}}\right)^{3} .
$$

The lamellar structure of the SDS micelle is stable, and has been used as a template for mesoporous metals. For example, the basal spacings of the metal film for $\mathrm{ZnO}$ were $d_{001}=3.1$ and 2.7 $\mathrm{nm}$, depending on the conditions. ${ }^{12,13}$ The separated SDS phase had a lamellar structure and the measured interlayer distance was $d_{001}=2.81 \mathrm{~nm}$ according to SAXS. The formation of a lamellar structure may be difficult for trivalent metals, because the steric strain is greater than for bivalent metals.

\section{Acknowledgements}

The present work was partially supported by a Grant-in-Aid for Scientific Research (No. 18510075) from the Japan Society for Promotion of Science (JSPS).

\section{References}

1. J. F. Scamehorn and J. H. Harwell, "Surfactant-Based Separations, Science and Technology", 2000, Chap. 10, ACS, Washington, D.C., 139.

2. D. L. Giokas, E. K. Paleologos, and M. I. Karayannis, J. Chem. Educ., 2003, 80, 61.

3. S. Tagashira, I. Fujiwara, K. Ochi, Y. Murakami, and Y. Sasaki, J. Environ. Chem., 2002, 12, 609.

4. Y. Murakami, K. Hiraiwa, Y. Sasaki, I. Fujiwara, and S. Tagashira, Anal. Sci., 2007, 23, 1147.

5. E. Kilinc, A. Cetin, M. Togrul, and H. Hosgoren, Anal. Sci., 2008, 24, 763.

6. T. Nakai, Y. Murakami, Y. Sasaki, and S. Tagashira, Talanta, $\mathbf{2 0 0 5}, 66,45$.

7. E. B. Sandell and H. Onishi, "Photometric Determination of Trace of Metals, General Aspects", 4th ed., 1978, John Wiley and Sons, New York, Chichester, 540.

8. S. Ide and M. Takagi, Anal. Sci., 1990, 6, 599.

9. P. Qin, C. Niu, G. Zeng, J. Zhu, and L. Yue, Anal. Sci., 2008, 24, 1205.

10. Y. Terada, A. Harada, K. Saito, S. Murakami, and A. Muromatsu, Bunseki Kagaku, 2003, 52, 725.

11. D. Myers, "Surfactant Science and Technology", 3rd ed., 2006, John Wiley and Sons, Hoboken, NJ, 112.

12. Y. Tan, E. M. P. Steinmiller, and K.-S. Choi, Langmuir, 2005, 21, 9618.

13. H. Luo, L. Sun, Y. Lu, and Y. Yan, Langmuir, 2004, 20, 10218. 